\title{
The 'tumour overkill syndrome'. A potentially lethal complication of cancer chemotherapy
}

\author{
M. L. SLEVIN \\ M.B.Ch.B., M.R.C.P.
A. M. COTTERILL
L.R.C.P., M.R.C.S.

R. BELL

M.B., F.R.A.C.P.

T. A. LISTER

M.D., M.R.C.P.

\begin{abstract}
Imperial Cancer Research Fund, Department of Medical Oncology, St Bartholomew's Hospital, London EC1A $7 B E$
\end{abstract}

\begin{abstract}
Summary
Two cases of acute leukaemia of Burkitt type in whom chemotherapy was associated with the 'tumour overkill syndrome' are described. One patient died with severe metabolic derangement which followed chemotherapy. In the other, an intensive programme of allopurinol administration, alkalinization of the urine and forced diuresis was instituted before chemotherapy, thus correcting the initial biochemical abnormalities and modifying those which occurred after chemotherapy. This approach prevented a fatal outcome, allowing the patient to survive to achieve bone marrow remission.
\end{abstract}

\section{Introduction}

The 'tumour overkill syndrome' (Bell et al., 1979) is a complex of biochemical abnormalities characterized by hyperuricaemia, hyperkalaemia, hyperphosphataemia, hypocalcaemia, metabolic acidosis and acute renal failure, that may occur in patients receiving chemotherapy for malignant disease. This syndrome occurs in patients with bulky tumours that are rapidly lysed following cytotoxic chemotherapy. This has been seen predominantly in patients with Burkitt's lymphoma and acute lymphoblastic leukaemia, but has also been reported in lymphocytic lymphoma and acute histiocytic lymphoma (Bell et al., 1979; Brereton et al., 1975; Cadman, Lundberg and Bertino, 1977; Muggia, Chia and Mickley, 1974; Zusman, Brown and Nesbit, 1973). Once established, the syndrome is difficult to reverse, and is often fatal.

\section{Case 1}

A 24-year-old housewife presented to her local hospital with intestinal obstruction. At laparotomy the findings were of small bowel intussusception with a tumour at the apex of the intussuscepted segment. There were numerous enlarged lymph nodes, but the liver and spleen appeared normal. Postoperative recovery was unremarkable. Histological examination of the excised tumour showed a Burkitt's lymphoma, and she was transferred to St Bartholomew's Hospital 10 days after surgery. On examination she was pale and unwell. The liver was enlarged $5 \mathrm{~cm}$ and the spleen $6 \mathrm{~cm}$ below the costal margin. There was generalized lymphadenopathy and bilateral breast masses were palpated. The $\mathrm{Hb}$ was $10 \cdot 6 \mathrm{~g} / \mathrm{dl}$, the $\mathrm{WCC}$ was $8 \times 10^{9} / \mathrm{l}$ and the bone marrow was infiltrated with Burkitt-like lymphoblasts. There was no evidence of liver or renal dysfunction. The uric acid was slightly raised at $0.5 \mathrm{mmol} / \mathrm{l}$. The patient was treated with adriamycin $25 \mathrm{mg} / \mathrm{m}^{2}$ i.v. on days 1 and 2 , and cyclophosphamide $1 \mathrm{~g} / \mathrm{m}^{2}$ i.v., vincristine $2 \mathrm{mg}$ i.v. and prednisolone $1 \mathrm{~g} / \mathrm{m}^{2}$ i.v. on day 2. Allopurinol $300 \mathrm{mg} /$ day was started on day 1. Twelve hours after therapy, the patient was tachypnoeic and had not voided urine. Catheterization showed only $200 \mathrm{ml}$ of turbid urine. Intravenous fluids and diuretics failed to establish a urine output. The biochemical values before and following chemotherapy are shown in Fig. 1. Within a few hours of treatment, the patient developed the 'tumour overkill syndrome'. Bicarbonate infusion and resonium retention enemas were given, and calcium chloride was given for tetany. The patient suffered cardiopulmonary arrest with ventricular fibrillation and died.

\section{Case 2}

A 26-year-old housewife presented with a 2-week history of dyspnoea and spontaneous bleeding. On admission, she was pale, with extensive purpura and ecchymoses. There was bilateral axillary lymphadenopathy. Masses $10 \times 10 \mathrm{~cm}$ were palpable in both breasts. The liver was enlarged $14 \mathrm{~cm}$ and the 

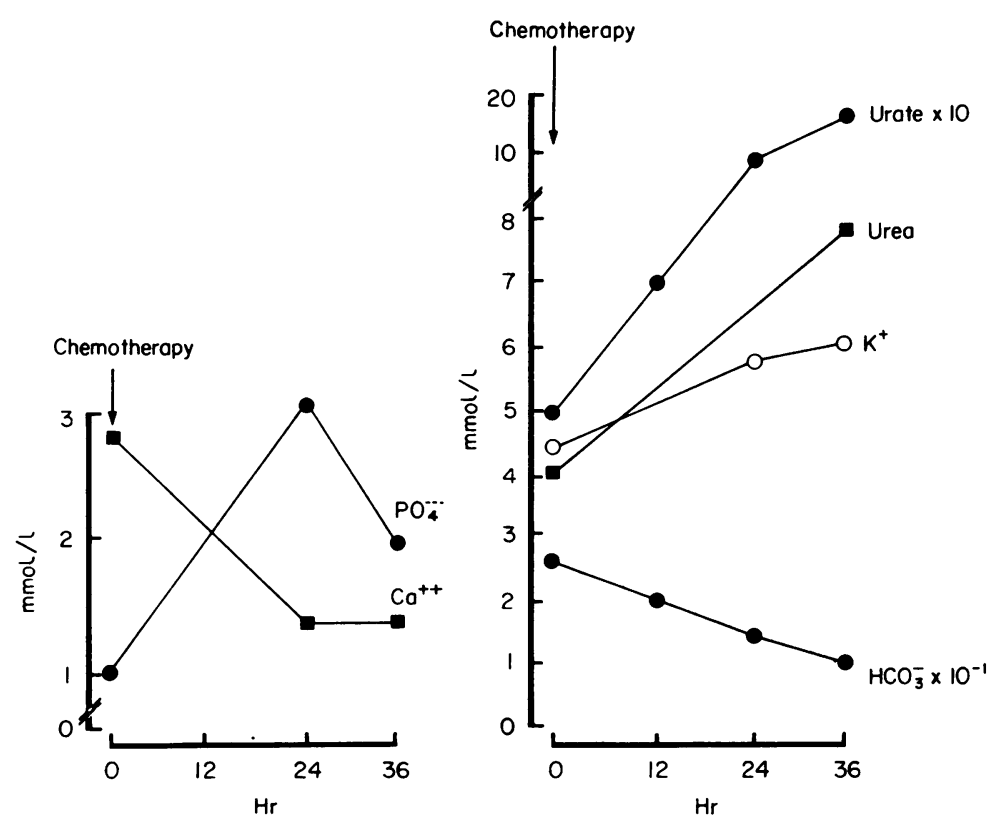

Fig. 1. Biochemical parameters before and during treatment of Case 1.

spleen $18 \mathrm{~cm}$ below the costal margin. The $\mathrm{Hb}$ was $7 \cdot 7 \mathrm{~g} / \mathrm{dl}$, the WCC $16 \cdot 1 \times 10^{9} / 1$ with $39 \%$ Burkitt-like lymphoblasts. The platelet count was $33 \times 10^{9} / 1$ and the bone marrow heavily infiltrated with Burkitt-like lymphoblasts. She was severely hyperuricaemic, was acidotic, and had mild renal impairment (Fig. 2).

Chemotherapy was withheld for the first $48 \mathrm{hr}, 0$ during which time allopurinol $900 \mathrm{mg} /$ day was administered orally, and more than 7 litres of fluid

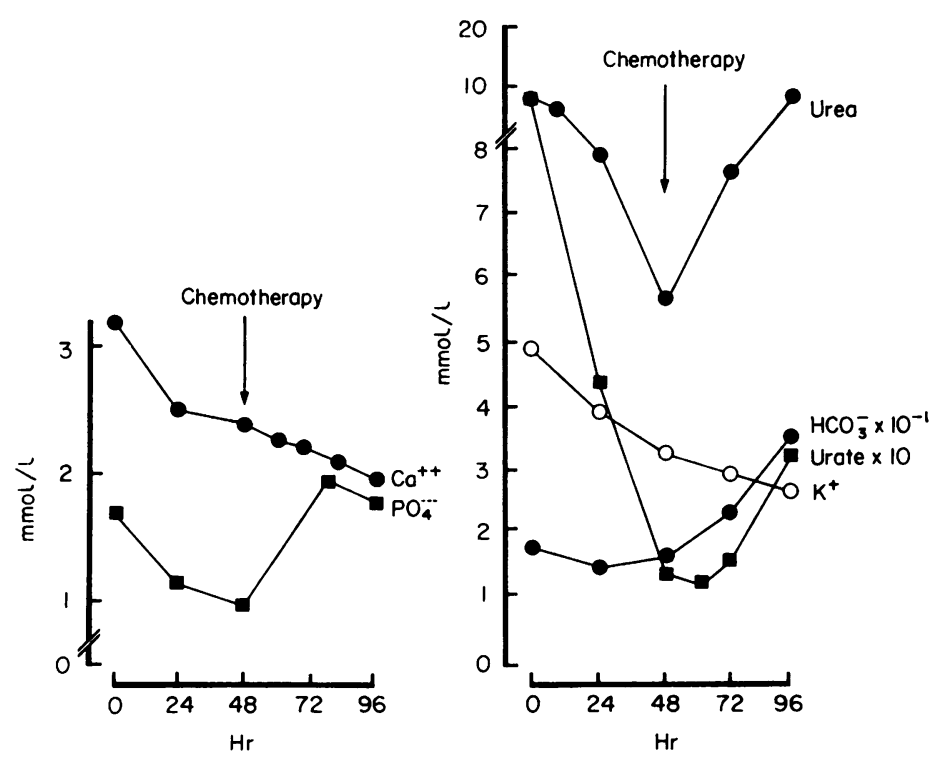

FIG. 2. Biochemical parameters before and during treatment of Case 2. 
daily were given i.v. and orally. Bicarbonate was given to maintain a urinary $\mathrm{pH}$ of 7 . This required $400 \mathrm{mEq}$ of $\mathrm{HCO}_{3} /$ day. Biochemical values on the day of admission, and the values on the morning of treatment, are shown in Fig. 2. The biochemistry was then considered to be optimal, and the patient was treated with modified doses of chemotherapy consisting of adriamycin $30 \mathrm{mg} / \mathrm{m}^{2}$ i.v. on day 1 , vincristine $2 \mathrm{mg}$ i.v. on day 1 and dexamethasone $2 \mathrm{mg} \times 4 /$ day. There was a massive reduction in all tumour masses within $24 \mathrm{hr}$. Mild features of the 'tumour overkill syndrome' became apparent with hyperphosphataemia and hypocalcaemia (Fig. 2). The serum urea increased slightly. On one occasion when the urinary $\mathrm{pH}$ fell below 7 , the urine became very opaque; however, this cleared when alkalinization was increased. With forced diuresis and large amounts of sodium bicarbonate, acidosis, hyperuricaemia and hyperkalaemia were not seen, and the patient survived. With 2 further cycles of chemotherapy bone marrow remission was achieved.

\section{Discussion}

The 'tumour overkill syndrome' can usually be anticipated as it tends to occur in patients with chemosensitive tumours and large bulky disease (Bell et al., 1979). In patient 1 , correction of the biochemical disturbance was only started after the patient had received chemotherapy and had developed the 'tumour overkill syndrome'. This proved to be too late and, despite vigorous therapy, she died. In patient 2 , intensive treatment for biochemical abnormalities before institution of chemotherapy corrected the initial metabolic disturbance and prevented the development of a severe "tumour overkill syndrome'. In order to withstand the large volumes of fluid and bicarbonate required to correct the biochemical abnormalities patients must have normal cardiovascular and renal function.

In order to deal with this potentially lethal complication of cancer chemotherapy, it is important to be aware of the circumstances in which it may occur, and that therapy be instituted either before chemotherapy, or at an early stage in the development of the syndrome.

\section{Acknowledgments}

The authors thank Dr John Amess in the Department of Haematology at St Bartholomew's Hospital for the morphological diagnosis, and the staff of the Department of Chemical Pathology for the multiple biochemical analyses performed.

They also thank Mrs Joanna Barton who helped prepare this manuscript.

\section{References}

Bell, R., Forbes, I.L., Sullivan, J.R. \& Hurley, T.H. (1979) Complications of tumour overkill when associated with high dose methotrexate therapy. Clinical and Experimental Pharmacology and Physiology, 5 (Suppl.), 47.

Brereton, H.D., Anderson, T., Johnson, R.E. \& Schein, P.S. (1975) Hyperphosphatemia and hypocalcemia in Burkitt's lymphoma. Archives of Internal Medicine, 135, 307.

Cadman, E.C., Lundberg, W.B. \& Bertino, J.R. (1977) Hyperphosphatemia and hypocalcemia accompanying rapid cell lysis in a patient with Burkitt's lymphoma and Burkitt cell leukemia. American Journal of Medicine, 62, 283.

Muggia, F.M., ChiA, G.A. \& Mickley, D.W. (1974) Hyperphosphatemia and hypocalcemia in neoplastic disorders. New England Journal of Medicine, 290, 857.

Zusman, J., Brown, D.M. \& NeSbit, M.E. (1973) Hyperphophatemia, hyperphosphaturia and hypocalcemia in acute lymphoblastic leukemia. New England Journal of Medicine, 289, 1335. 